\title{
ELECTRONIC BAND STRUCTURE OF CUBIC HgS*
} \author{
AND W. SzUSZKIEWICZ \\ Institute of Physics, Polish Academy of Sciences \\ Al. Lotników 32/46, 02-668 Warszawa, Poland
}

E. Guziewicz, B. Kowalski, B.A. Oreowski, K. Dybko, B. Witkowska

\begin{abstract}
Reflectivity spectra of $\mathrm{Hg}_{1-x} \mathrm{Fe}_{x} \mathrm{~S}(x<0.04)$ and $\mathrm{HgSe}_{1-y} \mathrm{~S}_{y}(y<0.5)$ mixed crystals were measured in the vacuum ultraviolet energy range from 4 to $12 \mathrm{eV}$. Information about the electronic band structure of cubic modification of $\mathrm{HgS}$ resulting from the above data is analyzed and discussed.
\end{abstract}

PACS numbers: $71.20 . \mathrm{Fi}$

The electronic band structure of all II-VI compounds which crystallize in the sphalerite (zinc blende) structure has been investigated long time ago and is well known. The only exception could be the cubic modification of mercury sulfide $(\beta-\mathrm{HgS})$. The reason is that due to its metastable character and significant problems with the crystal growth the bulk big size crystals of $\beta$-HgS have never been obtained. The investigations of the physical properties of this material were limited to the studies of the thin evaporated slabs, very small size pure policrystals, greater, but containing high concentration of different impurities, natural policrystals or the mixed crystals with $\mathrm{Cd}, \mathrm{Zn}, \mathrm{Se}$ or Te. It has been verified that $\beta$ - $\mathrm{HgS}$ is a narrow-gap semiconductor with an inverted band structure [1]. However, the only attempt of the electronic band structure investigations in the whole Brillouin zone by means of the ultraviolet reflectivity measurements was limited to the studies of the good quality thin evaporated slabs [2]. The results of the $\beta$ - $\mathrm{HgS}$ band structure calculations have also been published $[3,4]$.

The recently grown big size cubic $\mathrm{HgS}$ crystals stabilized by mercury sulfide doping with transition metal impurities [5] opened new possibility in electronic band structure investigations of the material under consideration. It was possible to investigate trends resulting from the measurements performed on different mixed crystals grown on the basis of $\mathrm{HgS}$. For the reflectivity studies two systems have been chosen: semimagnetic semiconductor $\mathrm{Hg}_{1-x} \mathrm{Fe}_{x} \mathrm{~S}$ and the mixed crystal with two anions $\mathrm{HgSe}_{1-y} \mathrm{~S}_{y}$.

It is believed that the $\mathrm{S}$ solubility limit in $\mathrm{HgSe}$ is about $80 \%$, the Fe solubility limit in $\mathrm{HgS}$ is not known up to now. $\mathrm{Hg}_{1-x} \mathrm{Fe}_{x} \mathrm{~S}$ mixed crystals containing up to few percent of iron and $\mathrm{HgSe}_{1-y} \mathrm{~S}_{y}$ compounds in the composition range

*This work is supported by the following grants of the State Committee for Scientific Research (Republic of Poland): No. 2 P302 199 06, No. 204759101 and No. 204869101. 


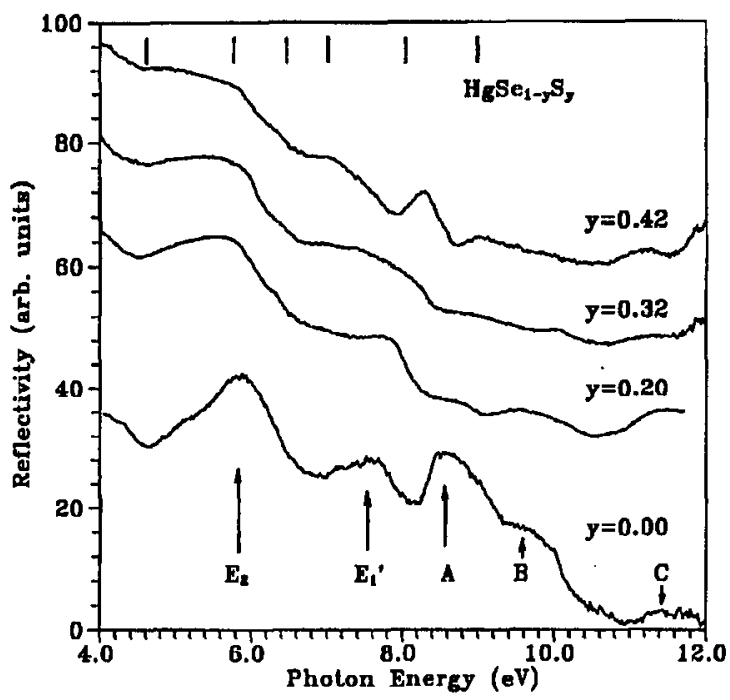

Fig. 1. Reflectivity spectra taken for the mixed crystals $\mathrm{HgSe}_{1-y} \mathrm{~S}_{y}$ at room temperature. The notation used for the structures corresponding to HgSe binary compound is shown. The marks in the upper part of the picture correspond to the energy positions of the most pronounced structures observed for the thin evaporated slabs of $\beta$-HgS [2].

$0<y<0.50$ were grown in the Institute of Physics, PAS, by a modified Bridgman method. All materials under investigations formed uniform solid solutions with the sphalerite crystal structure. The chemical composition was determined by scanning electron microscopy (SEM) microprobe, X-ray diffraction and energy dispersive X-ray fluorescence (EDXRF) method.

The reflectivity spectra were taken at room temperature for a vacuum ultraviolet radiation in the energy range from $4 \mathrm{eV}$ up to $12 \mathrm{eV}$. A vacuum monochromator based on the Seya-Namioka scheme was used, the source of the radiation was the hydrogen gas discharge lamp connected with the monochromator without any window. Other experimental details could be found in [6].

Figure 1 presents experimental spectra obtained for $\mathrm{HgSe}_{1-y} \mathrm{~S}_{y}$ with $y=0,0.20,0.32$ and 0.42 using the experimental setup mentioned above. Results of the similar reflectivity measurements performed for $\mathrm{Hg}_{1_{-}-x} \mathrm{Fe}_{x} \mathrm{~S}(x=0.01$ and 0.04) are shown in Fig. 2.

The following notation has been used for the particular transitions at selected high-symmetry points of $\mathrm{HgSe}$ Brillouin zone:

$-E_{2}$ maximum: transitions at $X$ point $\left(X_{6}^{\mathrm{v}}-X_{6}^{\mathrm{c}}\right.$ and/or $\left.X_{7}^{\mathrm{v}}-X_{7}^{\mathrm{c}}\right)$,

- $E_{1}^{\prime}$ maximum: transitions $L_{4,5}^{\mathrm{v}}-L_{4,5}^{\mathrm{c}}$ and $W_{6}^{\mathrm{v}}-W_{6}^{\mathrm{c}}$,

- $A$ maximum: transitions $L_{4,5}^{\mathrm{v}}-L_{4,5}^{\mathrm{c}}$ and $L_{4,5}^{\mathrm{v}}-L_{6^{\prime}}^{\mathrm{c}}$, band),

- $B$ maximum: transitions $L_{6}^{\mathbf{v}}$ (first valence band) $L_{6}^{\mathrm{c}}$ (third conduction band).

- $C$ maximum: transitions $L_{6}^{v}$ (second valence band) $L_{6}^{c}$ (third conduction 


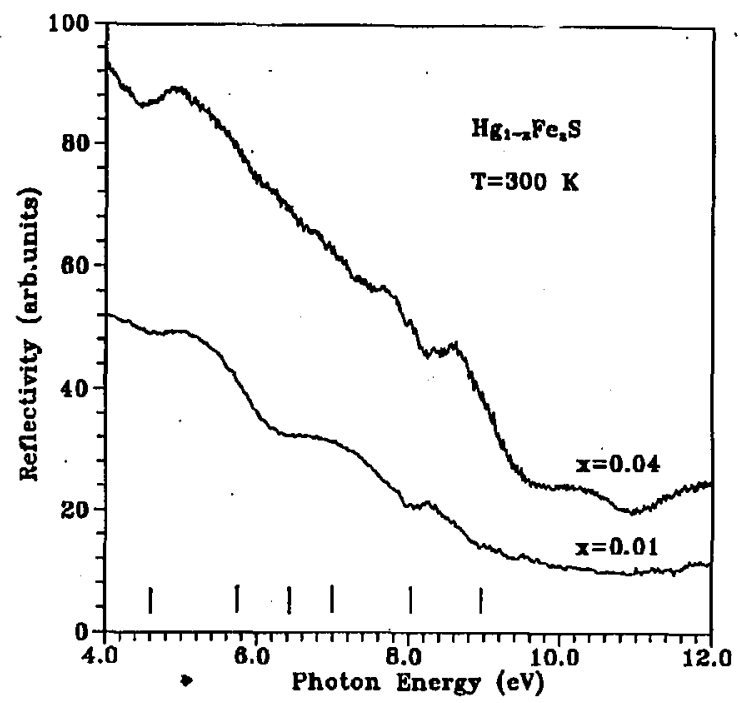

Fig. 2. Reflectivity spectra obtained for two $\mathrm{Hg}_{1-x} \mathrm{Fe}_{x} \mathrm{~S}$ mixed crystals with different chemical compositions. For the comparison with the data corresponding to pure $\beta-\mathrm{HgS}$ the same marks as these shown in Fig. 1 are also given.

As one can see from the experimental data taken for pure HgSe all five groups of optical transitions are well separated from each other. Moreover, the energies corresponding to different contributions to the same structure in the reflectivity spectrum do not differ noticeably. However, the reflectivity spectrum changes in a significant manner with the increasing amount of sulphur in the mixed crystal. The changes observed are not limited only to the shift in the energy positions of the structures. The results of theoretical calculations performed for cubic mercury sulphide $[3,4]$ are not as detailed as it has been done for $\mathrm{HgSe}$ and $\mathrm{HgTe}$. However, on the basis of identification of the optical transitions given in [2] one could expect for $\beta$-HgS substantial differences between the energies of optical transitions giving rise to the structures labeled as $E_{2}$ and $E_{1}^{\prime}$ in the case of $\mathrm{HgSe}$. The first effect results probably from the higher energy separations of the mercury sulphide valence bands in the $X$ high-symmetry point of the Brillouin zone than that observed for HgSe. The second effect perhaps demonstrates the significant difference between the energies of the appropriate transitions at $L$ and $W$ points. It was suggested in the literature that the high energy contributions to the $E_{2}$ and $E_{1}^{\prime}$ structures observed for $\beta$-HgS have the energies about $1 \mathrm{eV}$ higher than those observed in the reflectivity spectra taken for $\mathrm{HgSe}$ (energy values equal to $8.05 \mathrm{eV}$ and $8.95 \mathrm{eV}$, respectively, have been reported in [2]). On the contrary, it seems from the results of our measurements that the energies of optical transitions labeled as $A, B$ and $C$ are much more separated in the case of $\mathrm{HgSe}$ than it could be expected in the case of $\beta-\mathrm{HgS}$. This conclusion is consistent with the interpretation of the data shown in [2]. If the previously published and given here interpretations of the observed optical transitions are correct, the valence band of $\beta-\mathrm{HgS}$ is strongly modified in 
comparison to the valence bands of other mercury chalcogenides (both HgSe and $\mathrm{HgTe}$ ). The principal difference could be that for $\beta$-HgS at $L$ point of the Brillouin zone the energy separations between the valence bands are much smaller, and at $X$ point are much greater than those corresponding, e.g., to $\mathrm{HgSe}$. It should be stressed that the reflectivity data taken previously for $\mathrm{HgSe}_{1-x} \mathrm{Te}_{x}$ system [7] do not demonstrate the properties mentioned above.

It is well known that introduction of transition metal ions into II-VI compound causes considerable modification of the energy bands in some points of the Brillouin zone (see, e.g., [6]). In general, the presence of $\mathrm{Fe}$ ions leads both to a broadening of reflectivity peaks and to change of energy positions of selected peaks. The broadening of reflectivity structure can be treated as caused by disorder created due to the partial substitution of the host lattice $\mathrm{Hg}$ ions by Fe ions. As one can see from Fig. 2 the most pronounced effects observed for $\mathrm{Hg}_{1-x} \mathrm{Fe}_{x} \mathrm{~S}$ mixed crystal are the shift of the peak at energy just above $8.0 \mathrm{eV}$ into higher energies and the opposite shift of the broad peak at about $5.0 \mathrm{eV}$ with the increasing Fe amount. As we believe the first peak originates from the optical transitions at $X$ point of the Brillouin zone, the second one from the optical transitions at $L$ and $\Sigma$ points. Probably the peak observed at highest energies (appearing at about $10.3 \mathrm{eV}$ for the sample with $x=0.04$ ) is caused by the $B$ and $C$ type transitions (which contribute to the same structure in the reflectivity spectra). However, the proper description of the structures observed needs more experimental results obtained for higher values of $x$ and $y$. Such crystals have been recently grown, work is in progress and the conclusive results will be soon published. There exists also the clear need of the detailed theoretical calculations of the $\beta$-HgS electronic band structure.

\section{Acknowledgments}

Authors would like to express their gratitude to Prof. A. Mycielski for his interest in this work and valuable remarks.

\section{References}

[1] K.J. Siemsen, H.D. Riccius, Phys. Status Solidi 37, 445 (1970).

[2] H.D. Riccius, K.J. Siemsen, in: The Physics of Semimetals and Narrow-Gap Semiconductors, Eds. D.L. Carter, R.T. Bate, Pergamon Press, Oxford 1971, p. 493.

[3] F. Herman, R.L. Kortum, C.D. Kuglin, J.L. Shay, in: Proc. Int. Conf. II-VI Semiconducting Compounds, Providence, R.I., 1967, Ed. D.G. Thomas, W.A. Benjamin, New York 1967, p. 503.

[4] J.C. Phillips, J.A. van Vechten, Phys. Rev. B 2, 2147 (1970).

[5] W. Szuszkiewicz, B. Witkowska, M. Jouanne, Acta Phys. Pol. A 87, 415 (1995).

[6] A. Sarem, B.J. Kowalski, B.A. Orlowski, J. Phys. Condens. Matter 2, 8173 (1990).

[7] B.J. Kowalski, K. Dybko, A. Lemanska, B.A. Orlowski, Acta Phys. Pol. A 82, 845 (1992). 